\title{
Glycogen synthase kinase- $3 \beta$ regulates fractalkine production by altering its trafficking from Golgi to plasma membrane: implications for Alzheimer's disease
}

\author{
Almudena Fuster-Matanzo ${ }^{1,2,3} \cdot$ Jerónimo Jurado-Arjona $^{2,3} \cdot$ Stefano Benvegnù $^{2}$ • \\ Esther García $^{2}$ Patricia Martín-Maestro ${ }^{2,3} \cdot$ Raquel Gómez-Sintes $^{4} \cdot$ Félix Hernández $^{2,3}$. \\ Jesús Ávila ${ }^{2,3}$
}

Received: 8 August 2016/Revised: 17 October 2016/Accepted: 2 November 2016/Published online: 10 November 2016

(C) The Author(s) 2016. This article is published with open access at Springerlink.com

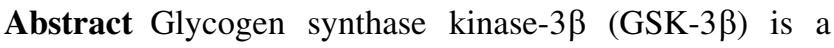
serine-threonine kinase implicated in multiple processes and signaling pathways. Its dysregulation is associated with different pathological conditions including Alzheimer's disease (AD). Here we demonstrate how changes in GSK$3 \beta$ activity and/or levels regulate the production and subsequent secretion of fractalkine, a chemokine involved in the immune response that has been linked to $\mathrm{AD}$ and to other different neurological disorders. Treatment of
\end{abstract}

A. Fuster-Matanzo and J. Jurado-Arjona contributed equally to this work.

Electronic supplementary material The online version of this article (doi:10.1007/s00018-016-2408-6) contains supplementary material, which is available to authorized users.

\footnotetext{
Almudena Fuster-Matanzo

af541@cam.ac.uk

$\triangle$ Jesús Ávila

jesus.avila@csic.es

1 Present Address: Department of Clinical Neurosciences, NIHR Biomedical Research Centre, Wellcome Trust-Medical Research Council Stem Cell Institute, University of Cambridge, Clifford Allbutt Building, Cambridge Biosciences Campus, Cambridge CB2 OPY, UK

2 Department of Molecular Neurobiology, Centro de Biología Molecular Severo Ochoa (CSIC-UAM), 28049 Madrid, Spain

3 Centro de Investigación Biomédica en Red sobre Enfermedades Neurodegenerativas (CIBERNED, ISCIII), Madrid, Spain

4 Departament of Cellular and Molecular Biology, Centro de Investigaciones Biológicas, CSIC, 28040 Madrid, Spain
}

primary cultured neurons with GSK-3 $\beta$ inhibitors such as lithium and AR-A014418 decreased full-length fractalkine in total cell extracts. Opposite effects were observed after neuron transduction with a lentiviral vector overexpressing the kinase. Biotinylation assays showed that those changes mainly affect the plasma membrane-associated form of the protein, an observation that positively correlates with changes in the levels of its soluble form. These effects were confirmed in lithium-treated wild type (wt) mice and in GSK-3 $\beta$ transgenic animals, as well as in brain samples from $\mathrm{AD}$ patients, evident as age-dependent (animals) or Braak stage dependent changes (humans) in both the membrane-bound and the soluble forms of the protein. Further immunohistochemical analyses demonstrated how GSK- $3 \beta$ exerts these effects by affecting the trafficking of the chemokine from the Golgi to the plasma membrane, in different and opposite ways when the levels/activity of the kinase are increased or decreased. This work provides for the first time a mechanism linking GSK- $3 \beta$ and fractalkine both in vitro and in vivo, with important implications for neurological disorders and especially for $\mathrm{AD}$, in which levels of this chemokine might be useful as a diagnostic tool.

Keywords GSK-3 3 - Fractalkine · Golgi network · Rab8 · Alzheimer's disease
Abbreviations
$\mathrm{AD}$
Alzheimer's disease
$\mathrm{A} \beta$
Amyloid $\beta$
APP
Amyloid precursor protein
Cdk-5
Cyclin-dependent-like kinase 5
GSK-3 $\beta$
Glycogen synthase kinase- $3 \beta$
GRASP 


\section{Introduction}

GSK-3 protein is a proline-directed serine/threonine protein kinase. In mammals there are two GSK-3 isoforms encoded by two different genes, GSK-3 $\alpha$ with a molecular weight of $51 \mathrm{kDa}$ and GSK-3 $\beta$ with a molecular weight of $47 \mathrm{kDa}$ [1]. GSK-3 can phosphorylate a variety of cytoplasmic and nuclear proteins and its substrates include cytoskeletal proteins, transcription factors and metabolic regulators. Thus, GSK-3 plays important roles both in embryonic development and in adulthood [2]. Dysregulation of GSK-3 activity is believed to play a key role in the pathogenesis of chronic central nervous system disorders, such as bipolar disorder, Huntington's disease and importantly in $\mathrm{AD}[3,4]$. Recently, different groups have documented that GSK3 $\beta$ activity is also crucial to regulate the inflammatory response by either promoting or inhibiting the process through the expression of pro- and antiinflammatory cytokines, respectively [5]. However, no connection between the kinase and fractalkine, an important chemokine in the inflammatory response, has been established so far.

Fractalkine, also known as neurotactin or CX3CL1, is an exceptional member of the large family of chemokines, with high expression levels in the brain [6]. Different cell types have been reported to express the protein including neurons [7]. Fractalkine exists in both soluble and membrane-associated forms. The $95 \mathrm{kDa}$ full-length protein is a type I transmembrane protein consisting of $\mathrm{N}$-terminal chemokine domain, a glycosylated mucin-like stalk, a transmembrane region and an intracellular C-terminal domain. The soluble form is approximately $70 \mathrm{kDa}$ and comprises the mucin-like stalk and the chemokine domain which can be released from the full-length protein via the action of several proteases, such as cathepsin S, ADAM10, and ADAM17 in both the periphery and the central nervous system [8-10]. Important functions have been described for both forms of the protein. While the membrane-anchored form acts as an adhesion molecule promoting retention of leucocytes to endothelial cells under physiological flow conditions [11], the soluble form exhibits chemotactic activity $[12,13]$. Fractalkine binds to a single receptor subtype, CX3CR1, expressed by myeloid cells including microglia [14], and interestingly, both soluble and transmembrane forms of the protein have been shown to ligate CX3CR1 [9, 10]. In the brain, this interaction controls microglia activation in basal conditions. However, depending on the type of injury, the fractalkine/CX3CR1 axis plays a different role in neurodegeneration versus neuroprotection [15]. Indeed, contradictory results have been obtained in different mouse models of $\mathrm{AD}[16,17]$. Thus, further studies are required to elucidate the implication of this chemokine-receptor interaction in the disease. In the context of mood disorders, the potential utility of fractalkine as a pathologically relevant biomarker or therapeutic target has been recently proposed for future clinical and translational research [18].

Considering the different roles played by the transmembrane and the soluble forms of fractalkine in the inflammatory response, it is of great importance to unravel the mechanisms responsible for the balance between both pools, particularly those regulating the availability of the chemokine at the plasma membrane which may directly influence the levels of the secreted form. Here we have identified GSK-3 $\beta$ as a novel regulator of fractalkine production and subsequent secretion, whose decreased activity or increased levels mediate opposite outcomes in the amount of both transmembrane and soluble forms in neurons. In line with this, we show for the first time that $\operatorname{Rab}^{+}$ vesicles are responsible for fractalkine transport from the trans Golgi to the plasma membrane, with GSK-3 $\beta$ being an active modulator of this transport. Interestingly, similar results regarding the levels of membrane-bound and soluble fractalkine were obtained in vivo with remarkable age dependent differences. Finally, a similar pattern was observed in brain samples from $\mathrm{AD}$ patients at different Braak stages. These findings reveal a novel and interesting GSK-3 $\beta$-mediated regulatory pathway for fractalkine, a chemokine that might be explored as a useful candidate for the early detection of AD.

\section{Materials and methods}

\section{Reagents}

The following reagents were used: AR-A014418 (Sigma), lithium chloride (Sigma), MG132 (Calbiochem), bafilomycin A1 (Sigma), Akt inhibitor VIII (Millipore), EZ-link Sulfo-NHS-LC-Biotin (Pierce), Dynabeads ${ }^{\circledR}$ M-280 Streptavidin (Thermo Fisher), complete Proteases Inhibitor (Roche), Lipofectamine 2000 (Invitrogen), DuoSet ELISA Kit for human samples and DuoSet ELISA Kit for mouse samples (R\&D Systems).

\section{Immunofluorescence and confocal microscopy}

Cells were fixed with $4 \%$ paraformaldehyde diluted in PBS at room temperature for $15 \mathrm{~min}$. They were permeabilized/ blocked with PBS/0.1\% Triton X-100/1\% (w/v) bovine serum albumin (BSA) for $1 \mathrm{~h}$. Subsequently, cells were incubated overnight at $4{ }^{\circ} \mathrm{C}$ with the following primary antibodies: anti-mouse Fractalkine 1/150 (R\&D systems), anti GM-130 1/200 (Abcam), anti TGN38 1/200 (NovusBio) and Rab8 1/200 (Santa Cruz Biotechnologies). After three washes with $1 \times$ PBS, cells were incubated for $1 \mathrm{~h}$ at 
room temperature with Alexa Fluor-conjugated secondary antibodies (Invitrogen), washed three times with $1 \times$ PBS and incubated with DAPI (Merck) for $10 \mathrm{~min}$. Stained cells were analyzed with a Zeiss LSM710 Vertical confocal microscope using a $63 \times$ objective and a 2.5 zoom. Single planes showing the greatest proportion of Golgi cisternae (either cis or trans) or $\mathrm{Rab}^{+}$vesicles were selected to acquire representative images.

\section{Animals}

\section{Animal care and treatments}

Mice were bred at the Centro de Biología Molecular and treated following the guidelines of Council of Europe Convention ETS123. GSK3 $\beta$ mice were generated as described previously [19]. Mice were kept on a normal light-dark cycle ( $12 \mathrm{~h}$ light/12 h dark), with free access to food and water. Male and female mice were used equally in all the experiments.

Two-month-old wild type C57BL/6 mice were fed with chow containing $1.7 \mathrm{~g} \mathrm{LiCl} / \mathrm{kg}$ (Harlan Teklad) for 2 weeks, followed by a diet containing $2.55 \mathrm{~g} \mathrm{LiCl} / \mathrm{kg}$ for 6 weeks. Control mice were fed with lithium-free chow under parallel conditions. Blood lithium levels were analyzed weekly by inductively coupled plasma mass spectrometry (ICP-MS). To prevent hyponatremia, water and a $\mathrm{NaCl}$ solution $(450 \mathrm{mM})$ were available ad libitum.

Three-month-old and 13-month-old GSK3 $\beta$ mice were used as representative groups of young and old animals, respectively.

\section{Human subjects}

The use of human brain tissue samples in this study was coordinated by the local Brain Bank (Banco de Tejidos CIEN, Madrid), following national laws and international ethical and technical guidelines on the use of human samples for biomedical research purposes [20]. In all cases, brain tissue donation, processing and use for research was performed in compliance with published protocols [21].

\section{Western blot}

Western blot analyses were prepared by homogenizing samples in ice-cold extraction buffer consisting of $50 \mathrm{mM}$ Tris $\mathrm{HCl}, \mathrm{pH} 7.4,150 \mathrm{mM} \mathrm{NaCl}, 1 \% \mathrm{NP}-40,1 \mathrm{mM}$ sodium orthovanadate, $1 \mathrm{mM}$ EDTA, a protease inhibitor cocktail and $1 \mu \mathrm{M}$ okadaic acid. Protein concentration was determined by a BCA Protein Assay kit (Thermo Scientific). $30 \mu \mathrm{g}$ of total protein were electrophoresed on $10 \%$ SDSpolyacrylamide gel and transferred to a nitrocellulose membrane (Schleicher \& Schuell, Keene, NH). The following primary antibodies were used: anti-mouse Fractalkine (R\&D systems), anti-human Fractalkine (R\&D systems), anti-PHF-1 (Tau P-Ser396/404) 1/100 (kind gift from Dr. Peter Davies, NY, USA), anti-Phospho-GSK- $3 \alpha / \beta$ (Ser21/9) (1/500) (Cell signaling), anti GSK-3 $\alpha / \beta(1 / 1000)$ (Cell signaling), anti-Phospho-Akt (Ser473) 1/500 (Cell Signaling), anti-Akt 1/1000 (Cell Signaling), anti- $\beta$ tubulin (1/5000) (Sigma), anti-GAPDH 1/10,000 (Abcam) and anti-flotilin-1 1/500 antibody (Abcam). Membranes were incubated with the antibody at $4{ }^{\circ} \mathrm{C}$ overnight in $5 \%$ nonfat dried milk. Secondary goat anti-mouse, anti-rabbit (1/1000; Invitrogen, San Diego, CA) and secondary donkey antigoat (1/1000 Santa Cruz Biotech) antibodies were used. ECL detection reagents (Amersham Biosciences, Arlington Heights, IL) were used for immunodetection. Quantification was performed with Image $\mathbf{J}$ software. Values were normalized with respect to the values obtained with an anti$\beta$-tubulin antibody for total extracts and with an anti-flotilin 1 antibody for membrane pellets to correct for total protein content.

\section{Statistical analysis}

A Kolmogorov-Smirnov test was performed for all data sets to test normality. When normal distribution was determined, comparisons between two independent samples were performed by a $t$ test and comparisons among groups were done by performing a one-way ANOVA. Nonnormal data sets were analyzed by equivalent nonparametric tests. $P<0.05$ values were considered significant. The SPSS v.22 software (SPSS, 1989; Apache Software Foundation) was used for all statistical analyses.

Additional methods are detailed in Supplemental information section.

\section{Results}

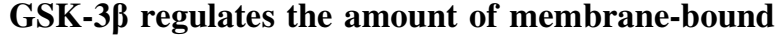 fractalkine with an impact on the levels of the soluble form}

Given the role of both GSK-3 $\beta$ and fractalkine in inflammatory processes and their implication in neurological disorders with a high inflammatory component, we wondered whether a possible link between the two proteins might exist. To investigate this, we first analyzed by Western blot, total extracts of primary cultured neurons treated with lithium, a classic general inhibitor of the kinase and with AR-A014418, a more specific inhibitor [22] (see Fig. S1). As it is shown in Fig. 1a, b, GSK-3 $\beta$ inhibition led to a dramatic decrease (around 50\%) in the levels of the mature membrane-bound form of fractalkine 

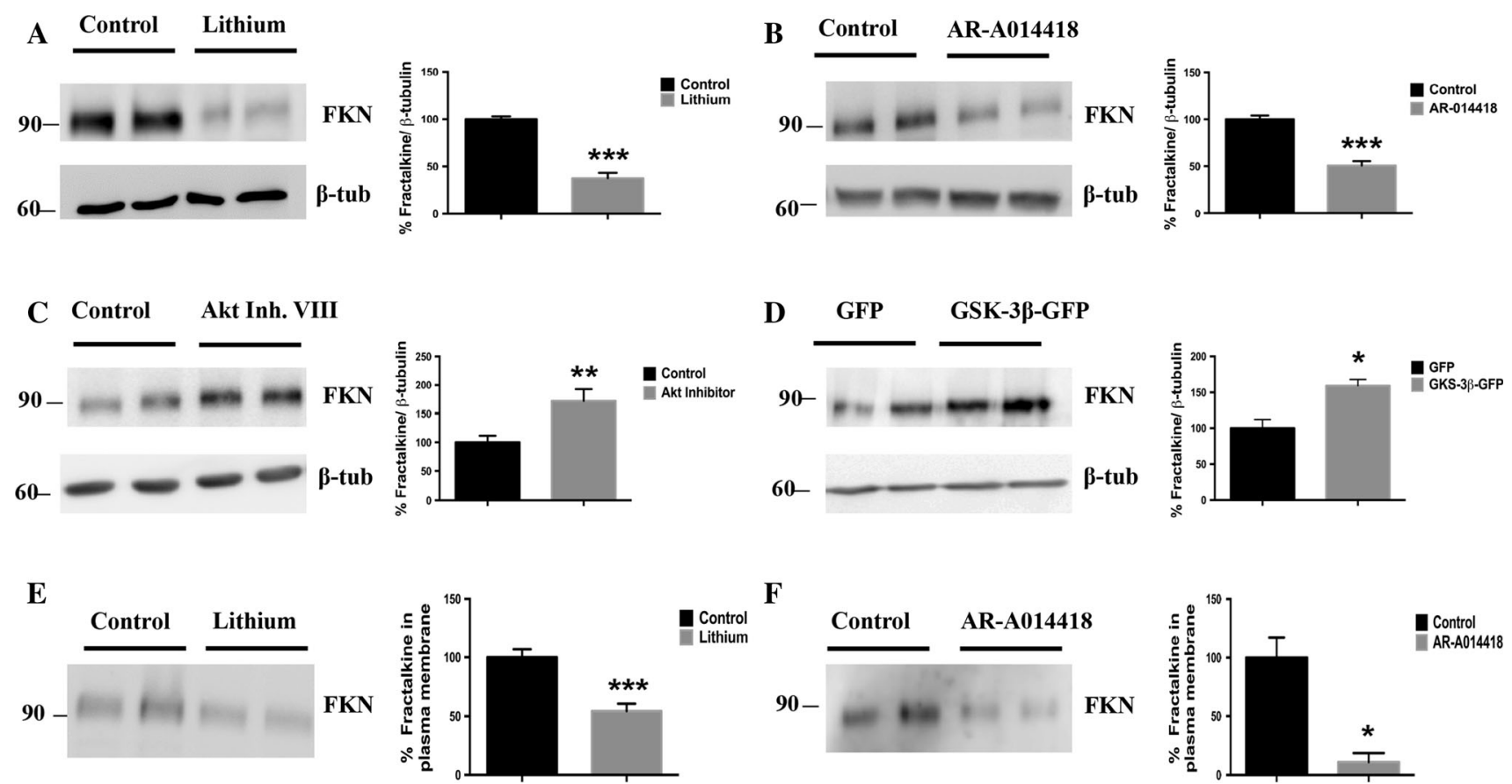

$\mathbf{F}$
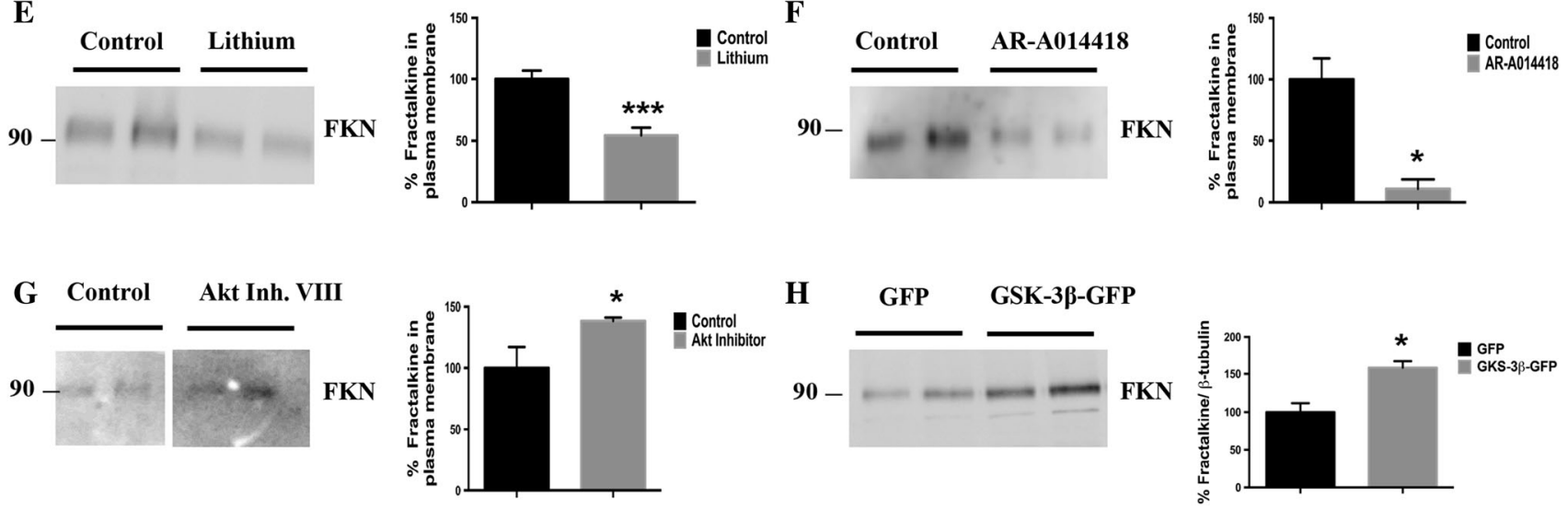
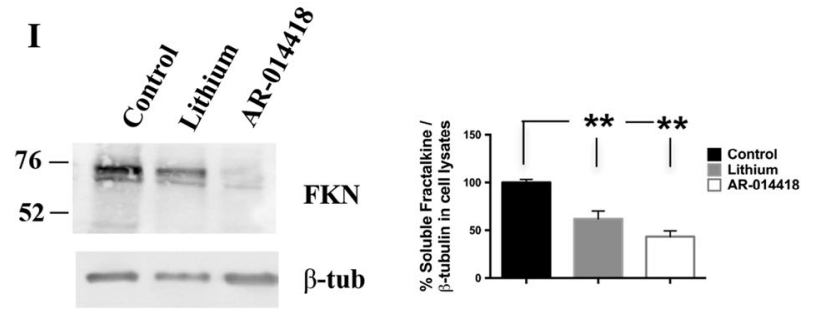

Fig. 1 Effects of GSK-3 $\beta$ on soluble and transmembrane fractalkine levels in vitro. Inhibition with lithium $(20 \mathrm{mM})$ (a) or AR-A014418 $(33 \mu \mathrm{M})(\mathbf{b})$ decreases total levels of fractalkine in neuronal lysates. GSK-3 $\beta$-increased activity by pharmacological approaches (Akt inhibitor VIII $(5 \mu \mathrm{M}))(\mathbf{c})$ or genetic approaches (lentiviral transduction) (d) increases total fractalkine levels in neuronal lysates. Biotinylation assays show how GSK-3 $\beta$-induced fractalkine changes mainly affect the membrane-bound form of the protein $(\mathbf{e}-\mathbf{h})$. Western

according to its molecular weight $(95 \mathrm{kDa})$. Opposite effects were detected when GSK-3 $\beta$ activity was increased by either a pharmacological approach through inhibition of Akt (a well-known upstream regulator of the kinase; Fig. 1c, Fig. S1) or by a genetic approach through neuron transduction with a GSK-3 $\beta$ overexpressing lentiviral construct (Fig. 1d, Fig. S1). In both cases, fractalkine

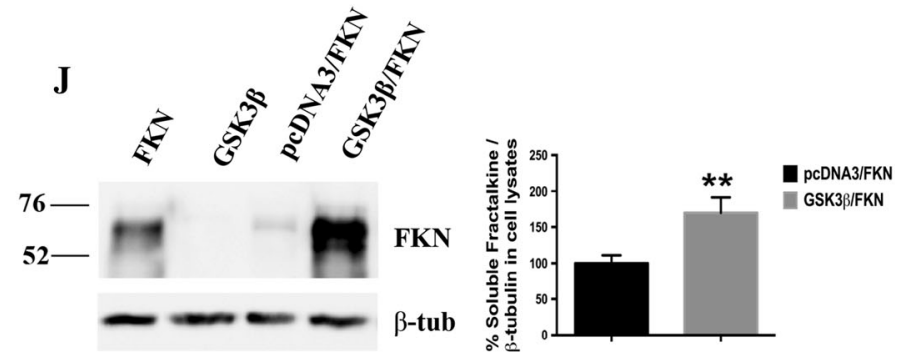

blot detection of fractalkine in concentrated media derived from transfected HEK293 cells treated with lithium $(20 \mathrm{mM})$ or ARA014418 $(33 \mu \mathrm{M})(\mathbf{i})$ or in co-transfection experiments $(\mathbf{j})$ showing a correlation with the observed changes in the membrane-anchored form. FKN, Fractalkine; $\beta$-tub, $\beta$-tubulin; Li, lithium; AR, ARA014418; Akt Inhib. VIII, Akt Inhibitor VIII. $* P<0.05$; $* * P<0.01 ; * * * P<0.001$ versus control/GFP/pcDNA3-FKN samples. $N \geq 3$ biological replicates. Data are expressed as mean \pm SEM

levels were found increased. These results pointed out to a regulation of fractalkine production mediated by the kinase. To clarify if the affected fractalkine pool was the membrane-bound one, we performed biotinylation assays. As it is observed in Fig. 1e-h, both inhibition and activation of GSK-3 $\beta$ (via pharmacological approach or lentiviral transduction) resulted in the same effects previously 
observed in total extracts, confirming that the kinase indeed affects the amount of the transmembrane form. Since fulllength fractalkine $(95 \mathrm{kDa})$ is cleaved at the plasma membrane to give rise to a soluble secreted form $(70 \mathrm{kDa})$ $[8,9]$, we wondered if GSK-3 $\beta$ induced changes in the membrane-bound pool could have an impact on the amount of soluble fractalkine. To address this, we analyzed media derived from fractalkine-expressing HEK293 cells treated with either lithium or AR-A014418, and from HEK293 cells co-expressing GSK-3 $\beta$ and fractalkine-expressing plasmids. Figure 1i, j shows how decreased fractalkine at the plasma membrane caused by GSK-3 $\beta$ inhibition is reflected in decreased levels of the soluble form, while increased levels of the membrane-bound form are translated into increased soluble fractalkine levels.

\section{GSK-3ß regulates Golgi-mediated fractalkine trafficking}

To obtain possible clues about the GSK-3 $\beta$-mediated mechanisms regulating fractalkine production and considering that the main protein degradation pathways were not responsible for the observed changes in fractalkine levels (Fig. S2), we performed an immunofluorescence assay in primary cultured neurons upon kinase inhibition/overexpression. Interestingly, in some neurons lithium and ARA014418 caused fractalkine signal to accumulate in the perinuclear region (Fig. S3A-C), a typical location for the Golgi apparatus, where indeed protein glycosylation and maturation processes normally take place [23]. Surprisingly, GSK-3 $\beta$ overexpression did not produce any remarkable change in fractalkine localization compared to GFP transduced control neurons (Fig. S3D, S3E). In an attempt to further characterize this possible Golgi-related phenomenon in GSK-3 $\beta$ inhibited neurons, we decided to perform colocalization experiments using typical markers of both cis and trans Golgi cisternae. The staining with the antibodies GM-130 and TGN-38 (cis and trans Golgi, respectively) revealed a fragmented pattern (Fig. S4A and Fig. 2a, respectively), something that had been previously described in HeLa cells [24]. However, in our model, this fragmentation preferentially affected the trans Golgi. Indeed, TGN38 staining revealed different fragmentation stages that in some cases, particularly corresponding to AR-A014418 treated neurons were more evident (Fig S5). We then quantified the percentage of positive GM-130 or TGN-38 vesicles that were also positive for fractalkine staining. Results regarding GM-130 did not reveal any difference between control and treated neurons (Fig. S4C, S4D). However, quantification with TGN-38 antibody resulted in a significant decreased colocalization in neurons treated with both inhibitors (Fig. 2c, d). In some cases, fractalkine staining gave the similar fragmented pattern observed with Golgi antibodies. In general, fragmentation was so severe that this fractalkine pattern was not observed anymore. These results confirmed that decreased GSK- $3 \beta$ activity was interrupting normal fractalkine processing due to Golgi disruption.

We next tried to find out if Golgi-related effects were also present in GSK-3 $\beta$ transduced neurons. To test that we stained GFP and GSK-3 $\beta$-GFP transduced neurons with GM-130 and TGN-38 antibodies, and we analyzed the percentage of colocalization with fractalkine antibody. Images and graphs in Figs. S4B and 2B show that in this case, increased GSK-3 $\beta$ levels did not produce any noticeable difference in Golgi morphology, but again decreased the percentage of fractalkine colocalization with trans Golgi antibody (Fig. 2e) without affecting its colocalization with GM-130 (Fig. S4E). These results together with the increased levels found in total extracts and in biotinylation experiments, might be indicative of a putative accelerated fractalkine trafficking from the trans Golgi network to the plasma membrane.

Rab GTPases are master regulators of the complex network of pathways that confirm the intracellular membrane traffic [25]. With the aim of testing the hypothesis of an accelerated fractalkine trafficking, we decided to pay attention to Rab8, a small GTPase that has been previously described to be involved in vesicular traffic between the trans Golgi cisternae and the plasma membrane [26]. As illustrated in Fig. 3a, a significant pool of Rab8 ${ }^{+}$vesicles colocalizes with fractalkine, confirming the implication of this GTPase in the transport of the protein, something that had not been described so far. We then performed another set of immunofluorescence assays, this time by analyzing the colocalization rates between Rab8 and fractalkine markers. Quantifications demonstrated that this percentage is lower in GSK-3 $\beta$ transduced neurons compared to the control (Fig. 3b). Furthermore, the total number of Rab ${ }^{+}$ vesicles under GSK-3 $\beta$ overexpression was also decreased (Fig. 3c). Together these results support the idea of an accelerated fractalkine trafficking as a consequence of an upregulated GSK-3 $\beta$ activity.

\section{GSK-3ß-mediated fractalkine changes are also observed in vivo}

Since our in vitro experiments demonstrated clear effects on both fractalkine membrane-bound form and fractalkine soluble form in cultured neurons mediated by GSK-3 $\beta$, we wondered if the same results would occur in mice. For this purpose, we first analyzed lithium-treated animals and collected membrane extracts and soluble enriched fractions from brain samples. Western blot analysis revealed that GSK-3 $\beta$ inhibition led to a $\sim 50 \%$ decrease in fractalkine levels (Fig. 4a, Fig. S6), which correlated with a 

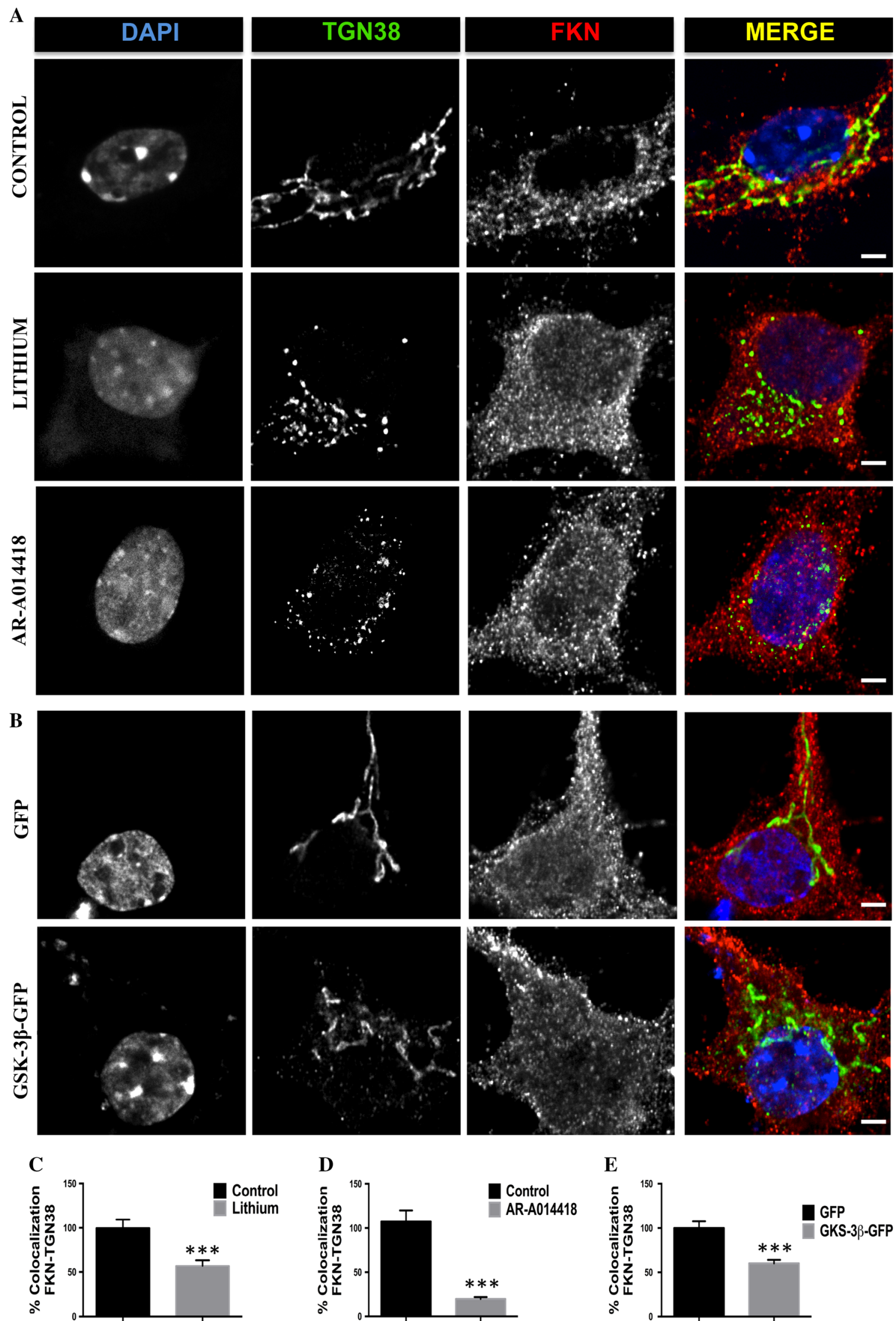

D

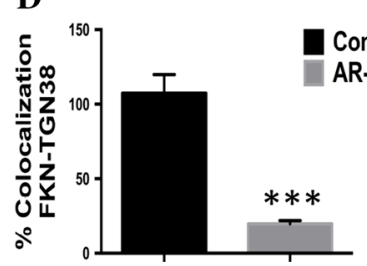

E

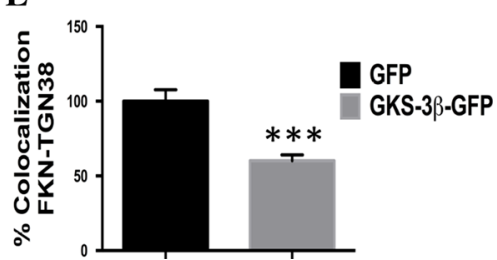


4Fig. 2 Effects of GSK-3 $\beta$ on fractalkine localization in the trans Golgi network. Immunostaining with trans Golgi marker TGN38 and fractalkine antibody showing an increased colocalization between both markers in neurons where the kinase was inhibited either by lithium $(20 \mathrm{mM})$ or AR-014418 $(33 \mu \mathrm{M})$ (a) and in neurons transduced with control GFP or GSK-3 $\beta$-GFP lentiviruses (b). Graphs representing the results of the colocalization analysis in treated (c, d) or transduced neurons (e). Right column in a and b shows merge images. $* P<0.05 ; * * P<0.01$ versus control. Scale bar $3 \mu \mathrm{m} . \quad N \geq 3$ biological replicates. Data are expressed as mean \pm SEM

concomitant decrease in the soluble form (Fig. 4b), confirming our previous in vitro results.

We next studied the same issue in a model with GSK-3 $\beta$ overexpression in the forebrain (Tet/GSK-3 $\beta$ mice) that was previously demonstrated to display an Alzheimer's disease-like phenotype [27]. Since the over-activation of the kinase has been reported to be a key factor in many neurodegenerative diseases in which some of the alterations show a temporal pattern [28], we decided to evaluate fractalkine levels in 3-month-old and 13-month-old mice. Interestingly, increased levels in membrane extracts were detected in both young (Fig. 4c) and old mice (Fig. 4e). However, only in 3-month-old mice, increased membrane levels positively correlated with increased soluble levels
(Fig. 4d), while in old mice no differences in the soluble enriched fractions were detected (Fig. 4f).

These results demonstrate how GSK- $3 \beta$ activity regulates membrane-bound fractalkine in vivo and how this is translated into changes in the soluble form of the protein only in young animals.

\section{Alzheimer's disease samples display similar fractalkine-related alterations}

Regarding the above-mentioned implication of GSK-3 $\beta$ in $\mathrm{AD}$, as well as the controversial role of fractalkine signaling in the disease, and taking into account our in vitro and in vivo results, we finally studied fractalkine levels in brain extracts from patients of different Braak stages (see Fig. S7). Importantly, we found a similar pattern to the one observed in Tet/GSK-3 $\beta$ mice. Thus, in early Braak II-III stages, increased levels of both membrane-associated (Fig. 5a) and soluble fractalkine (Fig. 5b) levels were found. Interestingly, in the later stages no statistical differences were detected in either membrane-bound (Fig. 5c) or soluble protein (Fig. 5d).

Altogether these results confirm a regulation of fractalkine in $\mathrm{AD}$ brains that might be due to the dysregulated activity of GSK-3 $\beta$ in these patients. Furthermore, these
A
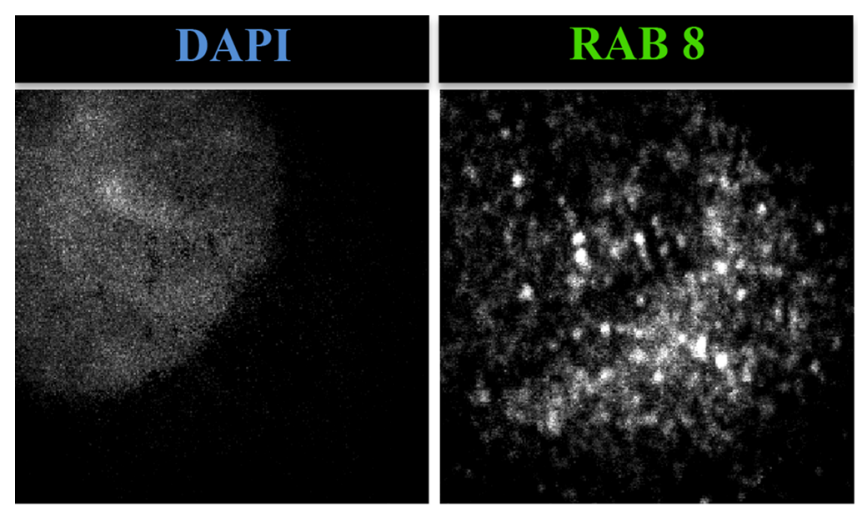

B

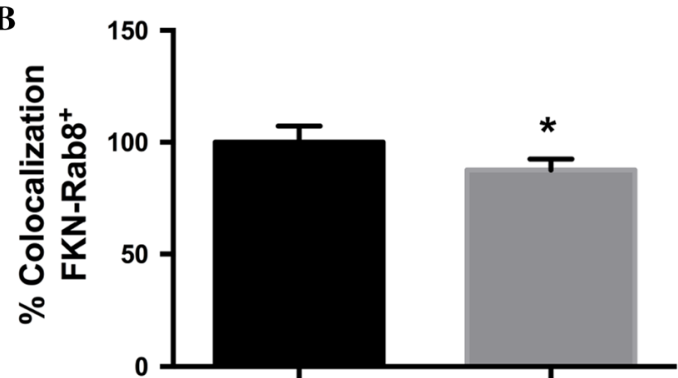

Fig. 3 GSK-3 $\beta$ overexpression affects fractalkine colocalization in $\mathrm{Rab}^{+}$vesicles. Immunostaining with Rab8 and fractalkine antibodies in GSK-3 $\beta$-GFP transduced neurons. Arrows show colocalization between both markers (a). GSK-3 $\beta$ decreases fractalkine

C
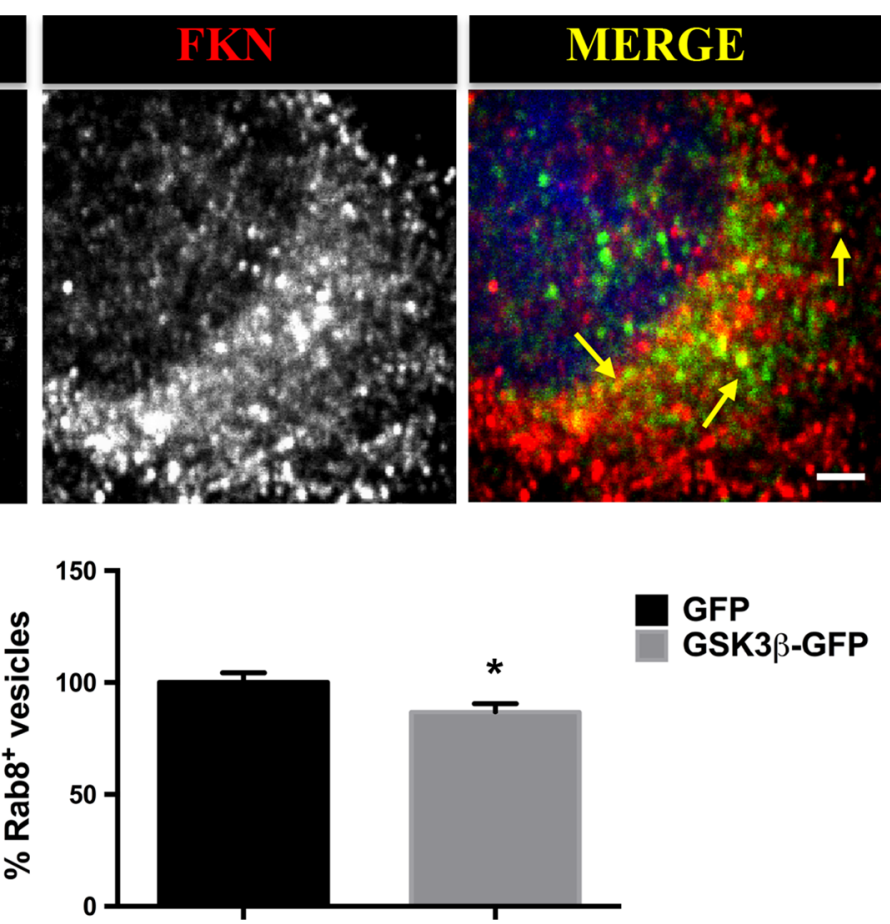

colocalization with $\mathrm{Rab}^{+}$vesicles (b). Total $\mathrm{Rab8}^{+}$vesicle pool is affected by GSK-3 $\beta$ upregulation $(\mathbf{c})$. ${ }^{*} P<0.05$ versus GFP transduced neurons. Scale bar $3 \mu \mathrm{m} . N \geq 3$ biological replicates. Data are expressed as mean \pm SEM 

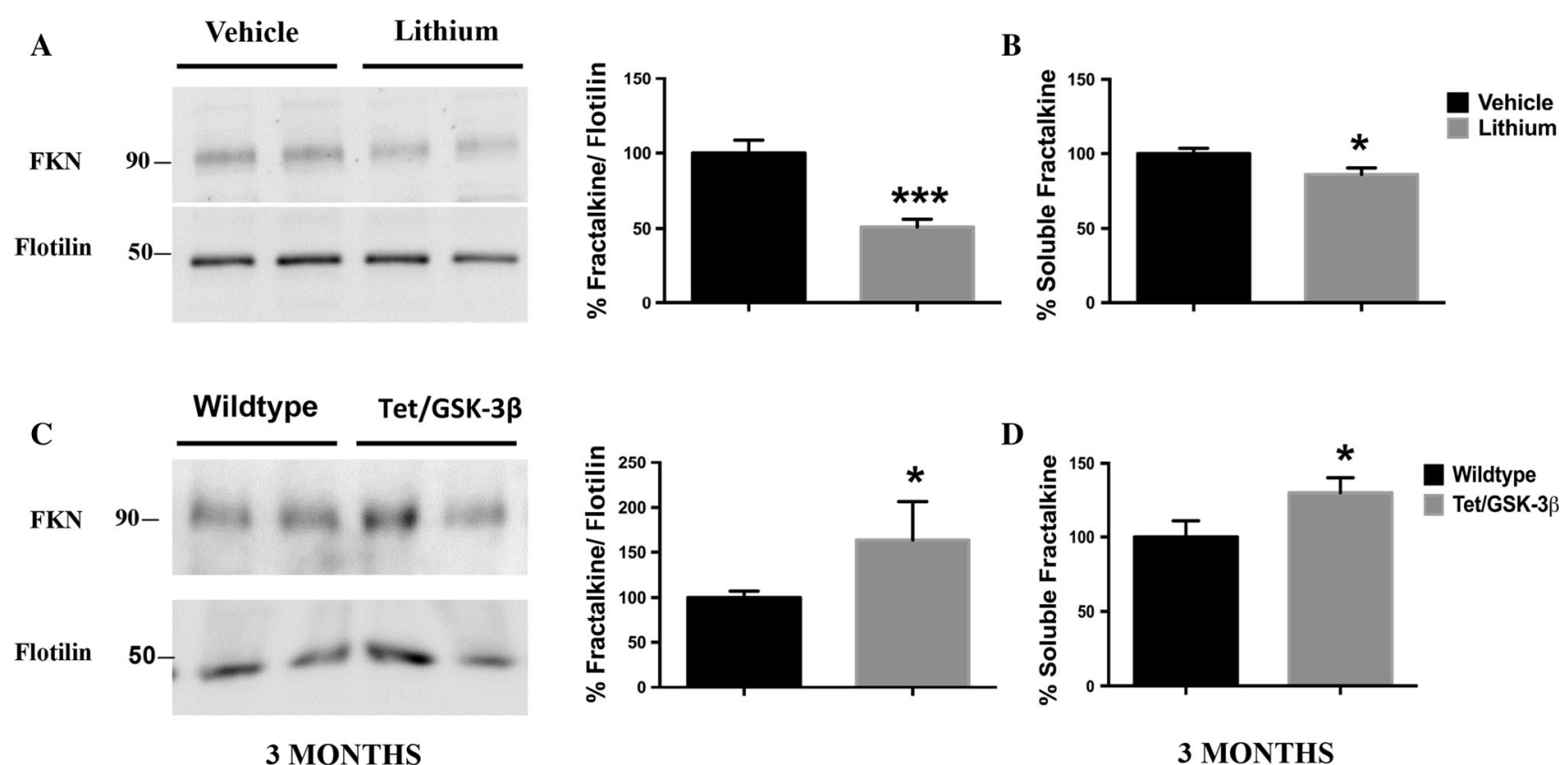

3 MONTHS

\section{MONTHS}

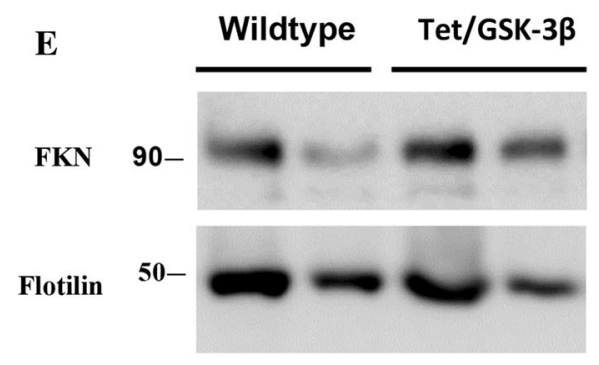

13 MONTHS

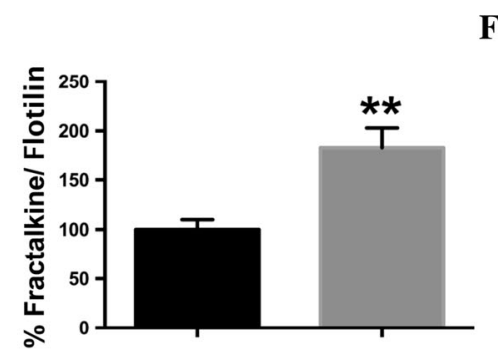

Fig. 4 Effects of GSK-3 $\beta$ on soluble and transmembrane fractalkine levels in vivo. GSK-3 $\beta$ inhibition also decreases fractalkine levels in membrane pellet extracts from wt animals treated with lithium (a). Tet/GSK-3 $\beta$ transgenic animals show increased fractalkine levels in membrane pellet extracts from 3-month-old animals (c) and 13-month-old mice (e). Graphs showing quantification of soluble fractalkine measured by ELISA in soluble enriched brain samples from wt animals treated with lithium (b) or 3-month-old (d) and

results strongly support the link between soluble fractalkine levels and AD progression, pointing to GSK-3 $\beta$ as an important modulator of this phenomenon during the disease.

\section{Discussion}

Several important roles have been attributed to fractalkine/ CX3CR 1 communication in both health and disease of the central nervous system, with most of the studies to date having paid special attention to the receptor. In this study, we demonstrate how the amount of both membrane-anchored and soluble fractalkine depends on GSK-3 $\beta$ activity/levels, a kinase which has been proposed as a key 13-month-old (f) Tet/GSK-3 $\beta$ animals. A correlation between the soluble fractalkine and the membrane-bound form levels was found in lithium-treated animals and young transgenic mice. $F K N$ fractalkine. $* P<0.05 ; * * P<0.01 ; * * * P<0.001$ versus control. Lithiumtreated mice, $N=9$ per group; 3-month-old transgenic animals, wt $N=13$, Tet/GSK-3 $\beta \quad N=10 ; 13$-month-old animals, $N=6$ per group. Data are expressed as mean \pm SEM

element in AD pathology [29] and with many other implications in other neurological disorders as well [28]. Our results indicate how GSK-3 $\beta$ regulates the availability of fractalkine at the plasma membrane, and as a result, the amount of the cleaved form in the medium, by interfering in the protein processing/maturation through the Golgi network.

The Golgi apparatus is a central intracellular membrane organelle for trafficking and modification of proteins and lipids which functions as a central hub in the exocytic secretory pathway [30]. Here, we have shown how modifications in GSK-3 $\beta$ activity/levels may impact in Golgimediated fractalkine trafficking in two different and opposite ways. First, in good agreement with the work of Adachi et al., our results demonstrate how Golgi 

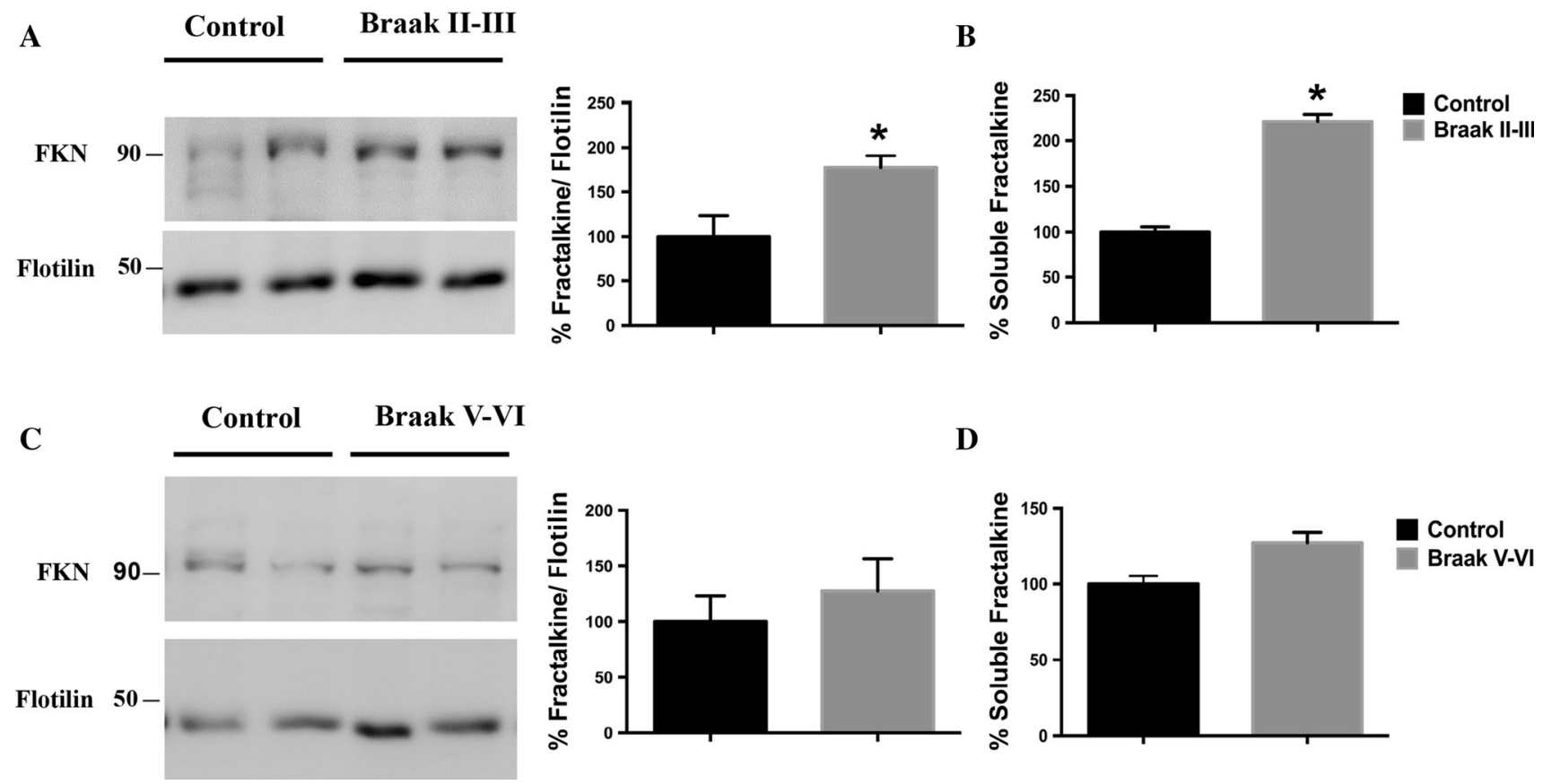

Fig. 5 Alzheimer's disease human samples also show altered transmembrane and soluble fractalkine levels. Fractalkine changes differ according to $\mathrm{AD}$ progression with increased levels in membrane pellet samples from II to III Braak stage (a) and no changes in V-VI Braak stage (c). Graphs representing the quantification of soluble fractalkine measured by ELISA in soluble enriched brain samples

morphology is largely affected by the decrease in GSK-3 $\beta$ activity which results in both cis and trans cisternae fragmentation [24]; although as our results demonstrate, this fragmentation preferentially affects the trans network in neurons, resulting also in decreased immunofluorescence colocalization with fractalkine antibody particularly affecting this portion of the structure. Specifically, regarding the TGN38 marker, we have been able to observe different fragmentation stages that may represent the sequential events followed by the neuron in response to GSK-3 $\beta$ inhibition. In the end, this phenomenon leads to a complete disaggregation and disappearance of the trans Golgi network, interrupting the normal exocytic pathway followed by fractalkine.

When the kinase levels and/or activity are increased, the opposite effects occur. While no changes were detected in either the morphology of the Golgi apparatus or in the colocalization assays with GM-130 marker, a decrease was observed with TGN38, pointing to specific effects related to the trans Golgi network. Considering that total fractalkine levels are increased, and specifically, levels corresponding to the membrane-bound form, we argue that GSK-3 $\beta$ overexpression might be affecting the sorting of the chemokine. Indeed, it is known that a portion of GSK$3 \beta$ localizes in the trans Golgi, where it interacts with some Golgi associated proteins such as p230 [24] and MACF1 showing increased fractalkine levels in II-III Braak stage samples (b) and no changes in V-VI Braak stage Alzheimer's disease patients (d). $F K N$ fractalkine. $* P<0.05 ; * * P<0.01 ; * * * P<0.001$ versus control. Total $N=19$ (see Fig. S7). Data are expressed as mean \pm SEM

[31]. However, effects have been mainly described in knock down experiments. In the opposite scenario, the one that we describe in this work, increased GSK-3 $\beta$ levels and activity would raise the phosphorylation status of its putative substrates that in turn, might affect the functioning of the organelle. For example, the oligomerization of the Golgi reassembly stacking proteins (GRASP proteins) is regulated by phosphorylation [32]. Furthermore, several studies highlight their importance in Golgi structure maintenance and architecture which is required for accurate glycosylation and sorting [33]. Although no interaction between GRASPs and GSK-3 has been reported so far, they constitute a good example of how phosphorylation of Golgi associated proteins may control the functioning of the organelle.

Our work also highlights for the first time that the small GTPase Rab8 contributes to fractalkine transport from the trans Golgi to the plasma membrane. We have first demonstrated how fractalkine colocalizes with $\mathrm{Rab}^{+}$ vesicles and how the total number of these vesicles is reduced when GSK-3 $\beta$ is overexpressed in neurons. Although further experiments are required to confirm a GSK-3 $\beta$-induced accelerated fractalkine trafficking from the Golgi apparatus to the plasma membrane, our results greatly support this hypothesis. In this scenario, accelerated trafficking would lead to an increased fusion rate of the 
$\mathrm{Rab}^{+}$vesicles to the plasma membrane, causing a decrease in their total number as well as in the colocalization between them and fractalkine protein, and consequently, leading to higher levels of the fractalkine membrane-bound pool. Finally, supporting this idea it has been previously reported that GSK-3 $\beta$ favors the exchange of GDP/GTP for Rab8 [34], something that would enhance the activity of this latter and might contribute to the abovementioned acceleration in this pathway.

Importantly, GSK-3 $\beta$ variations affect fractalkine levels also in animal models. This might be relevant on one hand, in the context of neuropsychiatric disorders, where lithium has been traditionally used as a therapeutic drug [35]. On the other hand, results in Tet/GSK-3 $\beta$ mice might be interesting for the early diagnosis of $\mathrm{AD}$. In these animals, age-related variations in the levels of both the membranebound and the soluble form of fractalkine correlate with what it is observed in $\mathrm{AD}$ samples from early and late Braak stages. We argue that the discrepancy in the results concerning the membrane-bound form in the late Braak stages comparing to the transgenic model might be due to the multifactorial aspect of the pathology [36]. Curiously, both in old animals and in Braak V-VI human samples, we do not observe any difference in fractalkine soluble levels. This could be explained by the decreased activity of some of the metalloproteases mediating fractalkine cleavage. Indeed, ADAM-10 has been suggested as a valuable target for the prevention/treatment of $\mathrm{AD}$, where mutations attenuating its activity and decreased levels have been described in the pathology [37]. Our results are also in good agreement with a paper published in 2008 , where the level of soluble fractalkine in plasma was significantly greater in the patients with mild to moderate $\mathrm{AD}$ than in the patients with severe AD [38]. As a whole, all these results might be easily interpreted taking into account the neuroprotective role that has been extensively attributed to fractalkine [39]. Thus, in the earliest stages of the disease, elevated levels of the chemokine could constitute a protecting response against the initial injury. With time and pathology progression, these levels would decrease becoming undifferentiated from control patients in the later stages.

In conclusion, our results stress the importance of finely balanced GSK-3 $\beta$ levels for fractalkine delivery and how the increase or the decrease in its activity result in opposite effects with direct consequences in the total levels of both the membrane-bound and the soluble form. These effects are also present in vivo as well as in AD brain samples. As a consequence, fractalkine might not be considered as a simple neuroprotective responsive element but as an interesting diagnostic marker for $\mathrm{AD}$ instead.
Acknowledgements This study was funded by grants from Spanish Ministry of Economy and Competitiveness (SAF 2006-02424, BFU2008-03980, BFU-2010-21507), Comunidad de Madrid (SAL/0202/ 2006), Fundación M. Botín, Centro de Investigación Biomédica en Red sobre Enfermedades Neurodegenerativas (CIBERNED, ISCIII), and an institutional grant from the Fundación R. Areces. Authors would like to thank Dr. Alberto Rábano (Neuropathology Department, CIEN Foundation, Madrid, Spain) for the human brain samples, people from Laboratory 122 from the Centro de Biología Molecular Severo Ochoa (CSIC-UAM), Madrid, Spain, for the constant help and technical support and Dr. Jayden A. Smith (Clinical Neurosciences Deparment, University of Cambridge) for kindly revising and correcting English grammar.

\section{Compliance with ethical standards}

Conflict of interest The authors declare that there are no conflicts of interest.

Open Access This article is distributed under the terms of the Creative Commons Attribution 4.0 International License (http:// creativecommons.org/licenses/by/4.0/), which permits unrestricted use, distribution, and reproduction in any medium, provided you give appropriate credit to the original author(s) and the source, provide a link to the Creative Commons license, and indicate if changes were made.

\section{References}

1. Woodgett JR (1990) Molecular cloning and expression of glycogen synthase kinase-3/factor A. EMBO J 9:2431

2. Jope RS, Johnson GV (2004) The glamour and gloom of glycogen synthase kinase-3. Trends Biochem Sci 29:95. doi:10.1016/j. tibs.2003.12.004

3. Beurel E, Grieco SF, Jope RS (2015) Glycogen synthase kinase-3 (GSK3): regulation, actions, and diseases. Pharmacol Ther 148:114. doi:10.1016/j.pharmthera.2014.11.016

4. Hernandez F, Nido JD, Avila J, Villanueva N (2009) GSK3 inhibitors and disease. Mini Rev Med Chem 9:1024

5. Leroy K, Yilmaz Z, Brion JP (2007) Increased level of active GSK-3beta in Alzheimer's disease and accumulation in argyrophilic grains and in neurones at different stages of neurofibrillary degeneration. Neuropathol Appl Neurobiol 33:43. doi:10.1111/j.1365-2990.2006.00795.x

6. Pan Y, Lloyd C, Zhou H et al (1997) Neurotactin, a membraneanchored chemokine upregulated in brain inflammation. Nature 387:611. doi:10.1038/42491

7. Imaizumi T, Yoshida H, Satoh K (2004) Regulation of CX3CL1/ fractalkine expression in endothelial cells. J Atheroscler Thromb 11:15

8. Garton KJ, Gough PJ, Blobel CP et al (2001) Tumor necrosis factor-alpha-converting enzyme (ADAM17) mediates the cleavage and shedding of fractalkine (CX3CL1). J Biol Chem 276:37993. doi:10.1074/jbc.M106434200

9. Hundhausen C, Misztela D, Berkhout TA et al (2003) The disintegrin-like metalloproteinase ADAM10 is involved in constitutive cleavage of CX3CL1(fractalkine) and regulates CX3CL1-mediated cell-cell adhesion. Blood 102:1186. doi:10. 1182/blood-2002-12-3775

10. Cook A, Hippensteel R, Shimizu S, Nicolai J, Fatatis A, Meucci O (2010) Interactions between chemokines: regulation of fractalkine/CX3CL1 homeostasis by SDF/CXCL12 in cortical neurons. J Biol Chem 285:10563. doi:10.1074/jbc.M109.035477 
11. Fong AM, Robinson LA, Steeber DA et al (1998) Fractalkine and CX3CR1 mediate a novel mechanism of leukocyte capture, firm adhesion, and activation under physiologic flow. J Exp Med 188:1413

12. Bazan JF, Bacon KB, Hardiman G et al (1997) A new class of membrane-bound chemokine with a CX3C motif. Nature 385:640. doi:10.1038/385640a0

13. Corcione A, Ferretti E, Bertolotto M et al (2009) CX3CR1 is expressed by human B lymphocytes and mediates CX3CL1 driven chemotaxis of tonsil centrocytes. PLoS One 4:e8485. doi:10. 1371/journal.pone.0008485

14. Harrison JK, Jiang Y, Chen S et al (1998) Role for neuronally derived fractalkine in mediating interactions between neurons and CX3CR1-expressing microglia. Proc Natl Acad Sci USA 95:10896

15. Ferretti E, Pistoia V, Corcione A (2014) Role of fractalkine/ CX3CL1 and its receptor in the pathogenesis of inflammatory and malignant diseases with emphasis on B cell malignancies. Mediators Inflamm 2014:480941. doi:10.1155/2014/480941

16. Lee S, Varvel NH, Konerth ME et al (2010) CX3CR1 deficiency alters microglial activation and reduces beta-amyloid deposition in two Alzheimer's disease mouse models. Am J Pathol 177:2549. doi:10.2353/ajpath.2010.100265

17. Bhaskar K, Konerth M, Kokiko-Cochran ON, Cardona A, Ransohoff RM, Lamb BT (2010) Regulation of tau pathology by the microglial fractalkine receptor. Neuron 68:19. doi:10.1016/j. neuron.2010.08.023

18. Stuart MJ, Baune BT (2014) Chemokines and chemokine receptors in mood disorders, schizophrenia, and cognitive impairment: a systematic review of biomarker studies. Neurosci Biobehav Rev 42:93. doi:10.1016/j.neubiorev.2014.02.001

19. Lucas JJ, Hernandez F, Gomez-Ramos P, Moran MA, Hen R, Avila J (2001) Decreased nuclear beta-catenin, tau hyperphosphorylation and neurodegeneration in GSK-3beta conditional transgenic mice. EMBO J 20:27. doi:10.1093/emboj/20.1.27

20. Martinez-Martin P, Avila J, Investigators ADRU (2010) Alzheimer Center Reina Sofia Foundation: fighting the disease and providing overall solutions. J Alzheimers Dis 21:337. doi:10. 3233/JAD-2010-101149

21. International Society for Biological and Environmental Repositories Biopreservation and Biobanking 2012. Collection, retrieval, and distribution of, bmf research (2008) Cell Preserv Technol 6. doi:10.1089/cpt.2008.9997

22. Bhat R, Xue Y, Berg S et al (2003) Structural insights and biological effects of glycogen synthase kinase 3-specific inhibitor AR-A014418. J Biol Chem 278:45937. doi:10.1074/jbc. M306268200

23. Wilson C, Venditti R, Rega LR, Colanzi A, D’Angelo G, De Matteis MA (2011) The Golgi apparatus: an organelle with multiple complex functions. Biochem J 433:1. doi:10.1042/ BJ20101058

24. Adachi A, Kano F, Tsuboi T, Fujita M, Maeda Y, Murata M (2010) Golgi-associated GSK3beta regulates the sorting process of post-Golgi membrane trafficking. J Cell Sci 123:3215. doi:10. $1242 /$ jcs.063941

25. Hutagalung AH, Novick PJ (2011) Role of Rab GTPases in membrane traffic and cell physiology. Physiol Rev 91:119. doi:10.1152/physrev.00059.2009

26. Huber LA, Pimplikar S, Parton RG, Virta H, Zerial M, Simons K (1993) Rab8, a small GTPase involved in vesicular traffic between the TGN and the basolateral plasma membrane. J Cell Biol 123:35

27. Engel T, Hernandez F, Avila J, Lucas JJ (2006) Full reversal of Alzheimer's disease-like phenotype in a mouse model with conditional overexpression of glycogen synthase kinase-3. J Neurosci 26:5083. doi:10.1523/JNEUROSCI.0604-06.2006

28. Lei P, Ayton S, Bush AI, Adlard PA (2011) GSK-3 in Neurodegenerative Diseases. Int J Alzheimers Dis 2011:189246. doi: $10.4061 / 2011 / 189246$

29. Hernandez F, Lucas JJ, Avila J (2013) GSK3 and tau: two convergence points in Alzheimer's disease. J Alzheimers Dis 33(Suppl 1):S141. doi:10.3233/JAD-2012-129025

30. Klute MJ, Melancon P, Dacks JB (2011) Evolution and diversity of the Golgi. Cold Spring Harb Perspect Biol 3:a007849. doi:10. 1101/cshperspect.a007849

31. Chen HJ, Lin CM, Lin CS, Perez-Olle R, Leung CL, Liem RK (2006) The role of microtubule actin cross-linking factor 1 (MACF1) in the Wnt signaling pathway. Genes Dev 20:1933. doi:10.1101/gad.1411206

32. Tang D, Wang Y (2013) Cell cycle regulation of Golgi membrane dynamics. Trends Cell Biol 23:296. doi:10.1016/j.tcb.2013.01. 008

33. Zhang X, Wang Y (2015) GRASPs in Golgi Structure and Function. Front Cell Dev Biol 3:84. doi:10.3389/fcell.2015.00084

34. Zhang B, Zhang T, Wang G et al (2015) GSK3beta-Dzip1-Rab8 cascade regulates ciliogenesis after mitosis. PLoS Biol 13:e1002129. doi:10.1371/journal.pbio.1002129

35. Diniz BS, Machado-Vieira R, Forlenza OV (2013) Lithium and neuroprotection: translational evidence and implications for the treatment of neuropsychiatric disorders. Neuropsychiatr Dis Treat 9:493. doi:10.2147/NDT.S33086

36. Iqbal K, Grundke-Iqbal I (2010) Alzheimer's disease, a multifactorial disorder seeking multitherapies. Alzheimers Dement 6:420. doi:10.1016/j.jalz.2010.04.006

37. Manzine PR, de Franca Bram JM, Barham EJ et al (2013) ADAM10 as a biomarker for Alzheimer's disease: a study with Brazilian elderly. Dement Geriatr Cogn Disord 35:58. doi:10. $1159 / 000345983$

38. Kim TS, Lim HK, Lee JY et al (2008) Changes in the levels of plasma soluble fractalkine in patients with mild cognitive impairment and Alzheimer's disease. Neurosci Lett 436:196. doi:10.1016/j.neulet.2008.03.019

39. Lauro C, Catalano M, Trettel F, Limatola C (2015) Fractalkine in the nervous system: neuroprotective or neurotoxic molecule? Ann N Y Acad Sci 1351:141. doi:10.1111/nyas. 12805 